\title{
Unique Co-Catalytic Behavior of Protic lonic Liquids as Multifunctional Electrolytes for Water Splitting
}

\author{
Tianhao Li, ${ }^{[a, b]}$ Xiaoyan Wang, ${ }^{[a, b]}$ Weiyong Yuan, ${ }^{*[a, b]}$ and Chang Ming Li ${ }^{[a, b]}$
}

\begin{abstract}
Hydrogen production from water splitting holds great promise for solving today's energy crisis but it is challenging to have high efficiency and low cost. For the first time, a protic ionic liquid (PIL), diethylammonium formate (DEAF) was used as a multifunctional electrolyte in water splitting, demonstrating a unique role of co-catalyst for the hydrogen evolution reaction (HER) with the highest current density $\left(21 \mathrm{mAcm}^{-2}\right.$ at $-0.5 \mathrm{~V})$ and the most positive onset potential $(-0.002 \mathrm{~V})$ in comparison to aprotic ILs and commonly used inorganic salts. Moreover, the concentration of PIL and temperature of electrolyte solution were optimized. The possible mechanism for the multifunctionality and high performance for water splitting using this PIL was proposed using X-ray photoelectron spectroscopy (XPS). The high performance for HER, simplicity and low cost for synthesis, and unique properties make DEAF highly promising as an electrolyte toward water splitting.
\end{abstract}

The wide use of fossil fuels has led to many environmental impacts, such as the generation of $\mathrm{CO}_{2}, \mathrm{CO}$ and NO.$^{[1]}$ Moreover, the price of fossil fuel and difficulty of fossil fuel extraction are increasing due to its non-sustainability. Therefore, hydrogen, considered as a clean and high-density energy source and carrier, has gained increasing attention in the past decades. ${ }^{[2,3]}$ Among hydrogen-generation techniques, it is no doubt that electrochemical/photoelectrochemcial water splitting is the cleanest and simplest due to utilization of renewable energy sources, no carbon emissions, operation in small and simple devices, and the high purity of the hydrogen obtained. ${ }^{[4]}$ However, electrolytes applied in water splitting cells are mostly inorganic salts with strong acids or bases. These electrolyte solutions are quite corrosive, which decrease the stability of the electrode materials, limit the catalysts applicable for water splitting, and compromise the safety of operation. ${ }^{[5]}$

[a] Dr. T. Li, X. Wang, Prof. W. Yuan, Prof. C. M. Li

Institute of Clean Energy \& Advanced Materials

Faculty of Materials and Energy

Southwest University

No.2 Tiansheng Road, Beibei

Chongqing 400715 (China)

E-mail:yuanweiyong@swu.edu.cn

[b] Dr. T. Li, X. Wang, Prof. W. Yuan, Prof. C. M. Li

Chongqing Key Laboratory for Advanced Materials and Technologies of Clean Energy

Southwest University

No.2 Tiansheng Road, Beibei

Chongqing 400715 (China)

E-mail:yuanweiyong@swu.edu.cn

$\square$ Supporting Information for this article is available on the WWW under http://dx.doi.org/10.1002/celc.201500458.
Ionic liquids (ILs), a class of non-molecular ionic solvents with low melting points $\left(<100^{\circ} \mathrm{C}\right)$, have great potential as electrolytes in water splitting due to their many unique physicochemical properties, such as nearly no measurable vapor pressure at room temperature, high conductivity, and high chemical stability. ${ }^{[6]}$ Currently, only a few studies using ionic liquids for water splitting have been reported. Imidazolium-based ILs had been initially introduced as electrolytes in hydrogen production by water electrolysis. ${ }^{[7-10]}$ Then, an ammonium-sulfonic-acid-based IL was utilized as the electrolyte and greater efficiencies were obtained. ${ }^{[11]}$ However, the viscosities of all these ionic liquids are much higher than that of the commonly used inorganic salts, which could greatly reduce the transportation rate of ions to influence the hydrogen production. Also, their synthesis is quite tedious and time-consuming, and the reactants used are not cheap. In addition, all these ILs and inorganic salts for water splitting have only been applied as electrolytes. To our knowledge, there is no report of using ILs as multifunctional electrolytes for significantly boosting watersplitting performance.

Protic ionic liquids (PILs) are an interesting subset of ILs easily produced through proton transfer from a Brønsted acid (organic or inorganic acid) to Brønsted base (amine) with low cost and a short reaction time. Compared to aprotic ionic liquids (AILs), PILs possess some unique electrolyte-related properties, including higher conductivity and much lower viscosity. ${ }^{[12,13]}$ In addition, the protonic feature allows PILs to be considered as proton sources for the hydrogen evolution reaction (HER). ${ }^{[14]}$ Also, the amines generated from PILs could coordinate with $\mathrm{Pt}_{1}{ }^{[15,16]}$ which is the most widely used catalyst with the highest activity and stability for HER, thus changing its electronic structure for possible promotion of the catalytic performance. Therefore, PILs could serve as both electrolyte and co-catalyst for hydrogen production. However, no one has exploited PILs as either electrolyte or co-catalyst for HER in water splitting.

In this work, diethylammonium formate (DEAF), a PIL, was investigated as multifunctional electrolytes for Pt-catalyzed water splitting for the first time. As a comparison, two AlLs were synthesized and applied as new electrolytes in a watersplitting cells as well (Scheme 1). The possible mechanism for the multifunctionality and high performance for water splitting was proposed based on the experimental results.

In traditional water-splitting studies, inorganic salts, such as $\mathrm{Na}_{2} \mathrm{SO}_{4}, \mathrm{NaCl}$ or $\mathrm{KCl}$, would be introduced as the electrolytes in acidic or alkaline solution. Other commonly used electrolytes, such as $\mathrm{NaOH}$ or $\mathrm{KOH}$, were not used since the $\mathrm{pH}$ of these solutions is quite different from that of the DEAF solution. Firstly, PIL (DEAF) and other two salts $\left(\mathrm{Na}_{2} \mathrm{SO}_{4}\right.$ and $\left.\mathrm{KCl}\right)$ were 


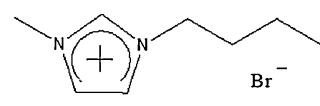

$\mathrm{BIMI-Br}$<smiles>CCNC[C+]C=O</smiles>

$\overline{\mathrm{Br}}$

BTAB

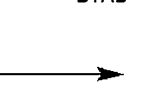<smiles>C[N+](C)(C)CCCCC=O</smiles>

DEAF<smiles>CC[NH2+]CC</smiles>

Scheme 1. Three ILs used in this study and synthesis of DEAF.

utilized as electrolytes for water-splitting experiments at the same $\mathrm{pH}$ and ionic strength.

As shown in Figure $1 \mathrm{~A}$, the linear sweep voltammogram (LSV) of the electrolytic cell using DEAF as the electrolyte exhibits a reduction peak with an onset potential at $-0.002 \mathrm{~V}$ $\left(E_{\text {onset }}\right)$ and a peak potential $\left(E_{\text {peak }}\right)$ at $-0.06 \mathrm{~V}$, which can be at-
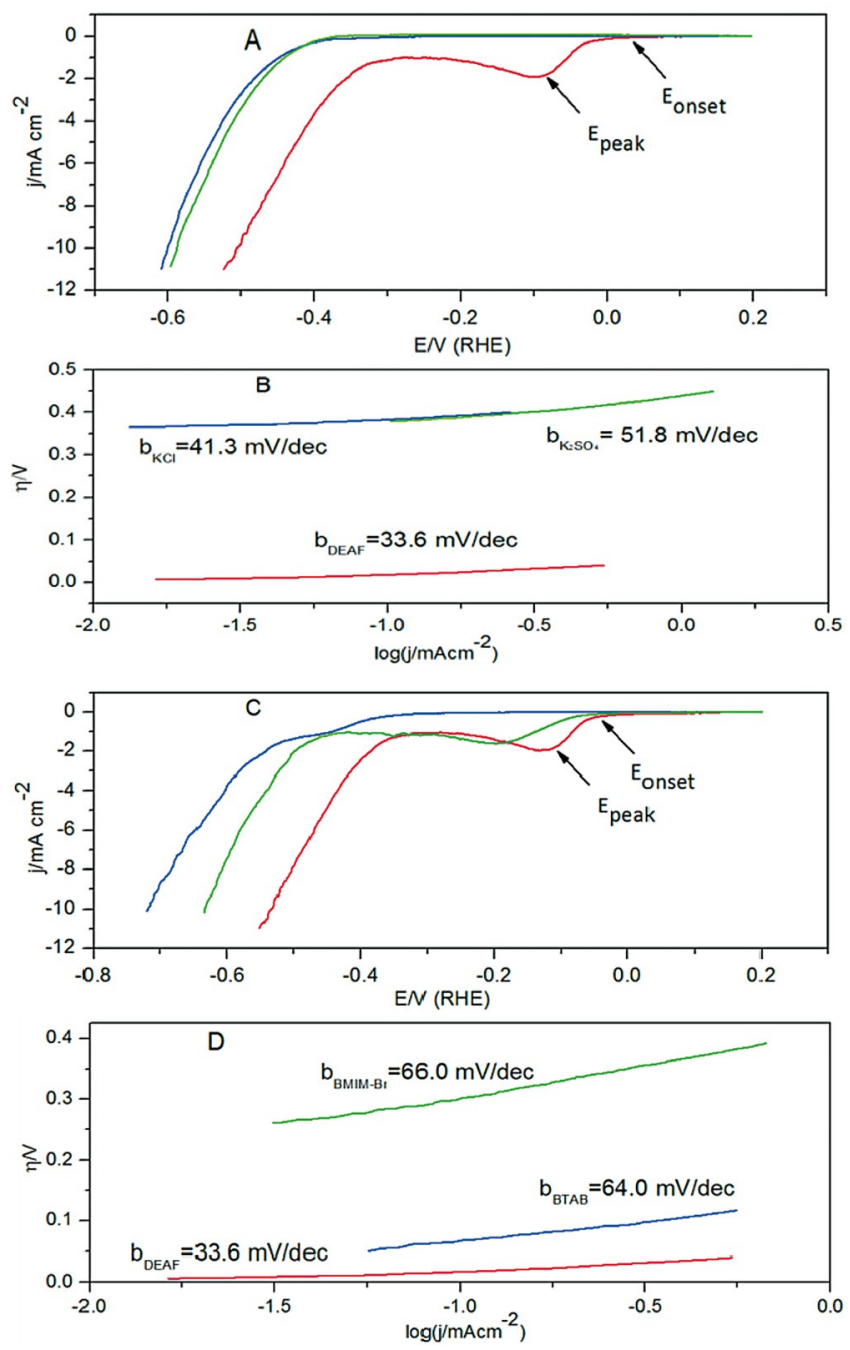

Figure 1. A) Linear sweep voltammograms at working electrode of different electrolyte solutions. Red: $1 \mathrm{M}$ DEAF, Blue: $1 \mathrm{M} \mathrm{KCl}$, Green: $0.33 \mathrm{M} \mathrm{K}_{2} \mathrm{SO}_{4}$. B) Tafel plots for HER using PIL and inorganic salts as electrolytes. Red: $1 \mathrm{M}$ DEAF, Blue: $1 \mathrm{M} \mathrm{KCl}$, Green: $0.33 \mathrm{M} \mathrm{K}_{2} \mathrm{SO}_{4}$. C) Linear sweep voltammograms at working electrode of different IL solutions. Red: $1 \mathrm{M}$ DEAF, Blue: $1 \mathrm{M}$ BTAB, Green: $1 \mathrm{M}$ BIMI-Br. D) Tafel plots for HER using PIL and aprotic ILs. Red: $1 \mathrm{M}$ DEAF, Blue: 1 м BTAB, Green: 1 м BIMI-Br. tributed to HER occurring on the $\mathrm{Pt} / \mathrm{C}$ catalyst. ${ }^{[7,17]}$ Compared to the cell using DEAF as the electrolyte, much more negative onset potentials for the cells using $\mathrm{Na}_{2} \mathrm{SO}_{4}$ and $\mathrm{KCl}$ as electrolytes were found at $-0.36 \mathrm{~V}$ and $-0.35 \mathrm{~V}$, respectively. Additionally, the current density of the DEAF-based cell is much higher than that of inorganic-salt-based cells at the same potential. It is worth noting that the $\mathrm{pH}$ of $1 \mathrm{M}$ DEAF is 4.6 , but that the other two neutral salt solutions need added $\mathrm{H}_{2} \mathrm{SO}_{4}$ for maintaining the same $\mathrm{pH}$ value. Thus, it is clear that the acidic PIL (DEAF) was not only used as electrolyte, but also applied as proton source without adding any other acids. Furthermore, Tafel plots for HER using these three electrolytes are displayed in Figure 1B, where the Tafel slopes $(b)$ were determined. Comparison of these slopes reveals that DEAF has the lowest Tafel slope, indicating that the kinetics for HER is improved using DEAF as the electrolyte. Thus, DEAF can interact with the catalyst to promote the catalytic performance.

To further understand the significant enhancement by using PIL as electrolyte for HER, BMIM-Br, a commonly used aprotic $\mathrm{IL}$, was used as the electrolyte and compared with DEAF under the same conditions. As shown in Figure $1 \mathrm{C}$, more positive onset and peak potentials ( $E_{\text {onset }}$ and $\left.E_{\text {peak }}\right)$ were obtained by using DEAF. Also, the current density was much larger than that using BMIM-Br. To confirm this finding, another aprotic IL (BTAB), which has a structure similar to DEAF but lacks the protonic feature, was applied as the electrolyte. Compared to DEAF, much more negative reduction potentials and smaller current densities were observed by using BTAB (Figure 1C). Moreover, Figure 1D shows that the Tafel slope of DEAF is much lower than that of the two aprotic ILs, which further suggests PIL could interact with catalyst to improve the HER kinetics. Clearly, besides the lower viscosity and proton source property of PIL which could improve the performance of water splitting ${ }^{[14,18]}$ the interaction between PIL and catalyst should be considered. It is noteworthy that there is an proton transfer equilibrium [Reaction (1)] in the PIL aqueous solution, where a small fraction of the PIL cations can deprotonate as proton source and regenerate the Brønsted base (the amine B). This amine could coordinate with Pt for enhancement of the catalytic activity:

$\left[\mathrm{BH}^{+}\right]\left[\mathrm{A}^{-}\right] \rightleftharpoons \mathrm{B}+\mathrm{HA}$

To explore this hypothesis, XPS was performed to identify the interaction between the PIL and catalyst (Figure 2). The binding energies (BEs) of $\mathrm{Pt}_{4} \mathrm{f}_{7 / 2}$ for the catatlyst and catalyst/ DEAF are 72.23 and $71.18 \mathrm{eV}$, respectively. This negative shift in the $\mathrm{BE}$ indicates an increase of electron density in the vincinty of $\mathrm{Pt}^{[19]}$ which should be due to the coordination between $\mathrm{Pt}$ and the diethyl amine generated from DEAF with lone-pair electrons. ${ }^{[15,16]}$ This increase in electron density will then enhance the affinity of protons in solution to the catalyst (Scheme 2). Therefore, PIL not only provides the proton for water splitting, but also generates the amines as a co-catalyst for enhancing the catalytic activity.

As DEAF was found to be the best electrolyte among the inorganic salts and ILs, experimental conditions, including PIL 


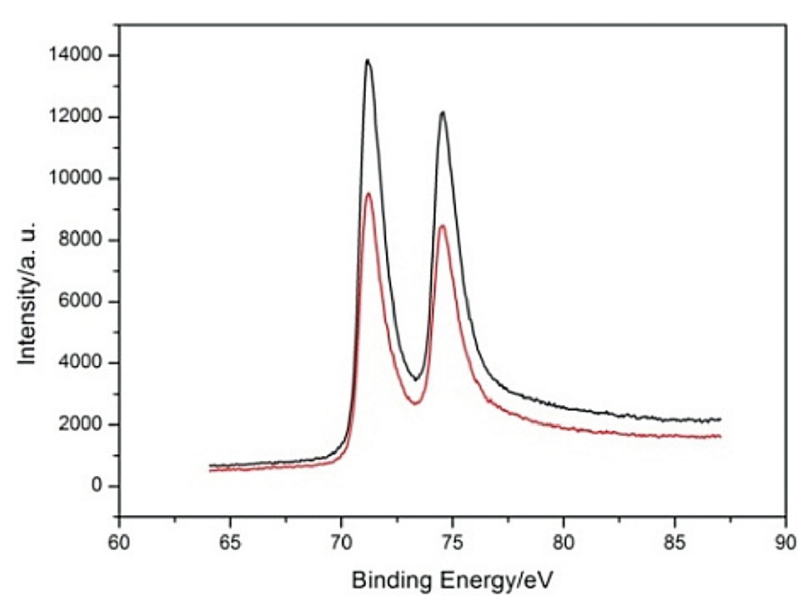

Figure 2. XPS spectra showing the Pt $4 \mathrm{f}$ doublets of the catalyst (Red) and catalyst/DEAF (Black).

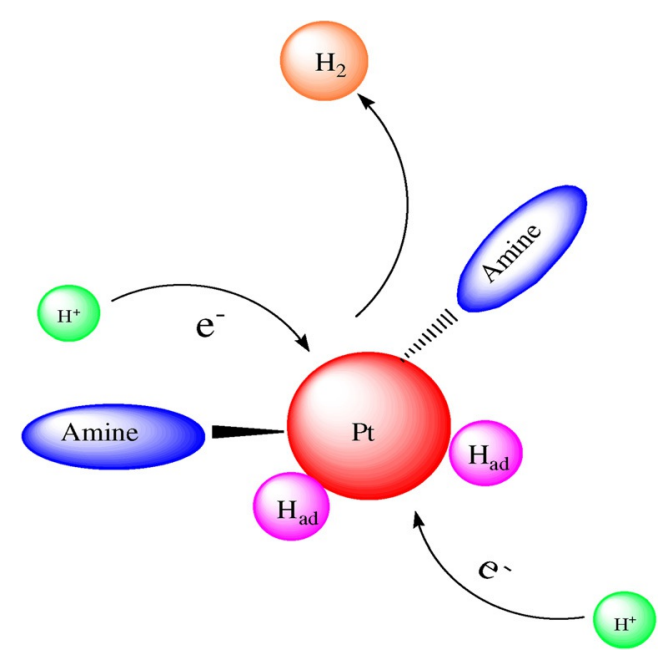

Scheme 2. Interaction between amine and catalyst.

concentration, temperature and working potential, were optimized. Initially, the effect of PIL concentration on the current densities $(j)$, onset potentials $\left(E_{\text {onset }}\right)$, and peak potentials $\left(E_{\text {peak }}\right)$ was evaluated by increasing the concentration of DEAF from 0.5 to $3.0 \mathrm{M}$. As shown in Figure $3 \mathrm{~A}$, the current densities recorded at $-0.5 \mathrm{~V}$ increase dramatically from -7.4 to $-13.3 \mathrm{mAcm}^{-2}$ as the concentration of DEAF increases from 0.5 to $1.5 \mathrm{M}$, which is due to the increase in solution conductivity and more amine groups involved in the interaction with $\mathrm{Pt}^{[18,20]}$ However, no significant variation in the current densities was observed when the PIL concentration is higher than $1.5 \mathrm{M}$. Moreover, Figure $3 \mathrm{~B}$ shows $E_{\text {onset }}$ decreasing gradually from -0.001 to $-0.012 \mathrm{~V}$ with the increase of PIL concentration. Similarly, $E_{\text {peak }}$ also changes from -0.06 to $-0.095 \mathrm{~V}$ (Figure $3 \mathrm{C}$ ). It can be attributed to the increase of solution viscosity with the increase of PIL concentration, ${ }^{[21]}$ which reduces the transportation rate of ions to deteriorate the hydrogen production performance. In an effort to minimize the influence of the viscosity and consumption of PIL while maintaining high cur- rent densities, a DEAF concentration of $1.5 \mathrm{M}$ was chosen for all subsequent studies.

The effect of temperature on current densities (j), onset potentials $\left(E_{\text {onset }}\right)$, and peak potentials $\left(E_{\text {peak }}\right)$ was also studied using a temperature ranging from 18 to $68^{\circ} \mathrm{C}$. As shown in Figure $3 \mathrm{D}$, the current density recorded at $-0.5 \mathrm{~V}$ increases significantly when the temperature increases from ambient to $48^{\circ} \mathrm{C}$, and then starts to decrease with further increase of the temperature. Similarly, the $E_{\text {onset }}$ and $E_{\text {peak }}$ slightly move to the most positive value and then gradually decrease with increasing temperature (Figures $3 \mathrm{E}, \mathrm{F}$ ). The increase in $j$ and potential should result from the faster diffusion of ionic species associated with a decrease of the solution viscosity when the solution is heated. ${ }^{[21]}$ However, the DEAF will start to decompose at high temperature, ${ }^{[22]}$ which could change the $\mathrm{pH}$ and decrease the conductivities of solution. Also, slightly sub-boiling conditions, as is usual in conventional electrolysis, did not work due to PIL decomposition. Therefore, the temperature range of 30$40^{\circ} \mathrm{C}$ could be probably the best working condition to compromise the water splitting performance while minimizing the decomposition of PIL and energy consumption.

Additionally, the working potential was optimized by analyzing the charge consumption $(Q)$ at the cathode with different potentials where the experiment was performed during a time period of $60 \mathrm{~min}$. As shown in Figure S1 (Supporting Information), no significant variation in $Q$ is obtained for a low potential $(E \geq-0.4 \mathrm{~V})$. Then, a dramatic increase of $\mathrm{Q}$ is observed when a higher potential was applied. Accordingly, $E \leq-0.5 \mathrm{~V}$ should be probably the best working water reduction potential.

The generated $\mathrm{H}_{2}$ was analyzed by gas chromatography under a constant potential. Only three measurements were conducted with 20 min duration between each one for each potential to obtain the general trend between the hydrogen generated and time. As shown in Figure 4, $47 \mu \mathrm{mol}$ of $\mathrm{H}_{2}$ was generated after an electrolysis period of $60 \mathrm{~min}$ with a Faradic efficiency of $94 \%$ as the potential was kept at $-0.48 \mathrm{~V}$. Moreover, a dramatic increase in the $\mathrm{H}_{2}$ production was observed when the potential increased.

The durability of the catalyst for HER using different electrolytes was preliminarily examined. The current densities and reduction potentials did not significantly change after a 30-hour experiment (approximate 200 times LSV) (Figure S2) and the $\mathrm{pH}$ of the electrolyte solution did not change, suggesting this PIL electrolyte did not deteriorate the activity of catalyst and that the PIL is quite stable during water splitting.

Herein, protic ionic liquid (PIL) was introduced as a multifunctional electrolyte in water splitting for the first time, demonstrating a unique role of co-catalyst for HER with the highest current density $\left(21 \mathrm{mAcm}^{-2}\right.$ at $\left.-0.5 \mathrm{~V}\right)$ and the most positive onset potential $(-0.002 \mathrm{~V})$ in comparison to the synthesized aprotic ILs and commonly used inorganic salts. Moreover, DEAF can be applied as a proton source without adding any other acid. With simplicity and low cost for synthesis, high performance for water splitting, and unique properties, DEAF is undoubtedly an outstanding multifunctional electrolyte for hydrogen production. More PILs are currently being investigated 

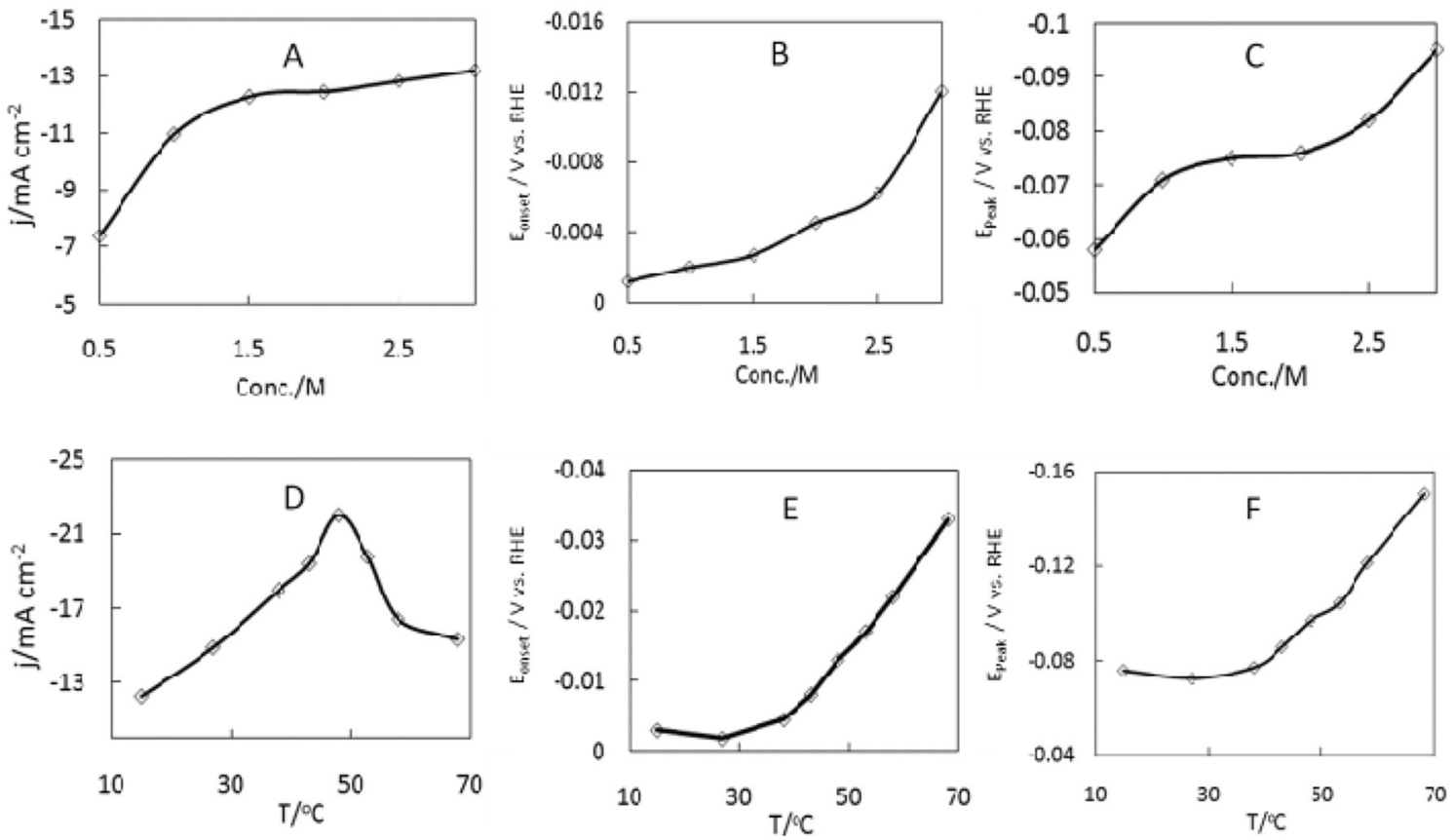

Figure 3. A) Effect of PIL concentration on the current densities (j) obtained by LSV at $-0.6 \mathrm{~V}$. B) Effect of PIL concentration on the $E_{\text {peak }}$ obtained by LSV. C) Effect of PIL concentration on the $E_{\text {onset }}$ obtained by LSV. D) Effect of temperature on the current densities (j) obtained by LSV at -0.6 V. E) Effect of temperature on the $E_{\text {peak }}$ obtained by LSV. F) Effect of temperature on the $E_{\text {onset }}$ obtained by LSV.

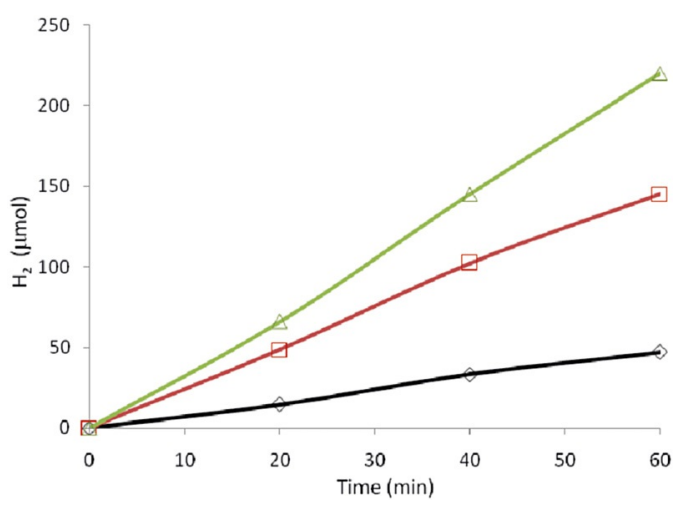

Figure 4. $\mathrm{H}_{2}$ production during 60 min with the working electrode applied potential during the water splitting of a PIL solution. Black: $-0.483 \mathrm{~V}$, Red: -0.583 V, Green: -0.683 V; Concentration of PIL: 1.5 M.

in our laboratory and more noble-metal-free catalysts will be applied to further study.

\section{Experimental Section}

1-butyl-3-methylimidazolium bromide (BMIM-Br), butyltriethylammonium bromide (BTAB) and diethylammonium formate (DEAF) were prepared and characterized according to literature reports. ${ }^{[23-25]}$ In comparison to other two AILs, DEAF can be easily synthesized via proton transfer from formic acid to diethyl amine with a dramatically short reaction time $(6 \mathrm{~h})$, and the crude product was simply purified under reduced pressure for $4 \mathrm{~h}$ at $50^{\circ} \mathrm{C}$.

The water-splitting experiments were performed on an electrochemical workstation (CHI-660E) in a three-electrode configuration comprised of a glassy carbon electrode (GCE) loaded with catalyst as the working electrode, a platinum plate and a saturated calomel electrode (SCE) as the counter electrode and the reference electrode, respectively. For preparation of the working electrode, $20 \mu \mathrm{L}$ of $1 \mathrm{mg} \mathrm{mL}^{-1}$ catalyst solutions with Nafion (mass ratio of Nafion to catalyst is 1 to 4) was dropped on the clean GC electrode and then dried. The catalyst is $30 \%(\mathrm{w} / \mathrm{w})$ commercial platinum carbon $(\mathrm{Pt} /$ C) purchased from Johnson Matthey. Three ILs and two inorganic salts were applied as electrolytes under the same conditions (ionic strength, $\mathrm{pH}$ and temperature). Since the $\mathrm{pH}$ of $1 \mathrm{M}$ DEAF solution was $4.6, \mathrm{H}_{2} \mathrm{SO}_{4}$ was added to other electrolytes solution, including $\mathrm{KCl}, \mathrm{K}_{2} \mathrm{SO}_{4}$ and other two ILs (BTAB and BIMI-Br), to obtained the same $\mathrm{pH}$. Then, the water-splitting system was investigated by varying concentration of PIL, temperature and working potential. The linear sweep voltammograms (LSV) were recorded at a scan rate of $2 \mathrm{mV} \mathrm{s}^{-1}$. Tafel plots for electrolytes (iR-corrected) were obtained by $\eta=b \log j+a$. Then, the corresponding Tafel slopes $(b)$ were used to determine the reaction kinetics. ${ }^{[26,27]}$ Chronoamperometry was carried out at a series of different potentials to obtain the charge consumption versus potential curves. All the tests were repeated at least three times. The potentials in all of the electrochemical measurements were converted to those with reference to a reversible hydrogen electrode (RHE). All reagents were supplied from Aladdin Chemistry Co. Ltd.

Hydrogen evaluation in $1.5 \mathrm{M}$ PIL electrolyte was detected in a closed system connecting to an Agilent Technologies 5890 gas chromatograph (GC) at a constant potential for $60 \mathrm{~min}$. After every 20 min of electrolysis under the $\mathrm{N}_{2}$ atmosphere, the headspace of electrolysis cell was collected and injected into the GC. The total amount of hydrogen was calculated over time.

X-ray photoelectron spectroscopy (XPS) of the catalyst and catalyst/PIL was performed using an ESCALAB 250Xi spectrometer under vacuum $\left(\sim 2 \times 10^{-9}\right.$ mbar). The excitation source was monochromatic Al Ka $(150 \mathrm{~W}, 1486.6 \mathrm{eV})$ radiation, and the C $1 \mathrm{~s}$ peak at $284.8 \mathrm{eV}$ was used as the reference for calibration. DEAF/catalyst samples were prepared by mixing the two components together. This mixture was filtered and washed by DI water for several times. 
Finally, the DEAF/catalyst sample was dried under vacuum for XPS analysis.

\section{Acknowledgements}

This work was supported by Fundamental Research Funds of the Central University (SWU114095, SWU114090, XDJK2015C027 and XDJK2015C026), Scientific Research Foundation for the Returned Overseas Chinese Scholars, Ministry of Education of China (Weiyong Yuan), and Chongqing Natural Science Foundation (No. cstc2015jcyjA50029).

Keywords: catalysts - hydrogen evolution reaction multifunctional electrolytes • protic ionic liquids • water splitting

[1] A. Ursua, L. M. Gandia, P. Sanchis, Proc. IEEE 2012, 100, 410-426.

[2] J. A. Turner, Science 2004, 305, 972-974.

[3] M. Z. Jacobson, W. G. Colella, D. M. Golden, Science 2005, 308, 1901 1905.

[4] R. L. Leroy, Int. J. Hydrogen Energy 1983, 8, 401-417.

[5] M. Wang, Z. Wang, X. Gong, Z. Guo, Renewable Sustainable Energy Rev. 2014, 29, 573-588.

[6] T. Welton, Chem. Rev. 1999, 99, 2071-2083.

[7] R. F. de Souza, J. C. Padilha, R. S. Gonçalves, J. Rault-Berthelot, Electrochem. Commun. 2006, 8, 211-216.

[8] R. F. de Souza, J. C. Padilha, R. S. Gonçalves, M. O. de Souza, J. Rault-Berthelot, J. Power Sources 2007, 164, $792-798$.

[9] R. F. de Souza, G. Loget, J. C. Padilha, E. A. Martini, M. O. de Souza, Elec trochem. Commun. 2008, 10, 1673-1675.

[10] G. Loget, J. C. Padilha, E. A. Martini, M. O. de Souza, R. F. de Souza, Int. J. Hydrogen Energy 2009, 34, 84-90.
[11] F. Fiegenbaum, E. M. Martini, M. O. de Souza, M. R. Becker, R. F. de Souza, J. Power Sources 2013, 243, $822-825$.

[12] M. A. B. H. Susan, A. Noda, S. Mitsushima, M. Watanabe, Chem. Commun. 2003, 938-939.

[13] J. Pernak, I. Goc, I. Mriska, Green Chem. 2004, 6, 323-329.

[14] M. H. Pool, M. P. Stewart, M. O'Hagan, W. J. Shaw, J. A. S. Roberts, R. M. Bullock, D. L. DuBois, PANS 2012, 109, 15634-15639.

[15] Y. Zou, B. Houten, N. Farrell, Biochemistry 1993, 32, 9632-9638.

[16] O. Pinato, C. Musertti, N. P. Farrell, C. Sissi, J. Inorg. Biochem. 2013, 122 , $27-37$

[17] U. Schröder, J. D. Wadhawan, R. G. Compton, F. Marken, P. A. Z. Suarez C. S. Consorti, R. F. Souza, J. Dupont, New J. Chem. 2000, 24, 1009 1015

[18] W. Xu, C. A. Angell, Science 2003, 302, 422-425.

[19] W. Yuan, Y. Cheng, P. K. Shen, C. M. Li, S. P. Jiang, J. Mater. Chem. A 2015, 3, $1961-1971$.

[20] A. P. Abbott, C. A. Eardley, J. Phys. Chem. B 2000, 104, $9351-9355$.

[21] G. Yu, D. Zhao, L. Wen, S. Yang, X. Cheng, AlChE J. 2012, 58, 2885 2899.

[22] T. L. Greaves, C. J. Drummond, Chem. Rev. 2008, 108, 206-237.

[23] K. Nishikawa, S. Wang, H. Katayanagi, S. Hayashi, H. Hamaguchi, Y. Koga, K. Tozaki, J. Phys. Chem. B 2007, 111, 4894-4900.

[24] H. Sakaebe, H. Matsumoto, K. Tatsumi, J. Power Sources 2005, 146, 693 697.

[25] T. L. Greaves, S. T. Mudie, C. J. Drummond, Phys. Chem. Chem. Phys. 2011, 13, $20441-20452$.

[26] J. Cheng, H. Zhang, G. Chen, Y. Zhang, Electrochim. Acta 2009, 54 6250-6256.

[27] J. Rossmeisl, Z. W. Qu, H. Zhu, G. K. Kores, J. K. Norskov, J. Electroanal Chem. 2007, 607, 83-89.

Manuscript received: October 20, 2015

Accepted Article published: November 17, 2015

Final Article published: November 25, 2015 